\title{
Reutilización de aguas residuales tratadas en la agricultura: una oportunidad para el sector cañero
}

Treated wastewater reuse in agriculture: an opportunity for the sugarcane industry

\section{JORGE ANTONIO SILVA-LEAL}

Ingeniero de Producción Biotecnológica Doctor en Ingeniería, Énfasis en Ingeniería Sanitaria y Ambiental Grupo de Investigación Gestión Ambiental Universidad de Boyacá, Colombia jorsilva@uniboyaca.edu.co

\section{PATRICIA TORRES-LOZADA}

Ingeniera Sanitaria

Doctora en Engenharia Hidraulica e Saneamento

Grupo ECCA

Universidad del Valle, Colombia

patricia.torres@correounivalle.edu.co

\section{CARLOS ARTURO MADERA-PARRA}

Ingeniero Sanitario

Mg. en Ingeniería Sanitaria y Ambiental

Grupo ECCA

Universidad del Valle, Colombia

carlos.a.madera@correounivalle.edu.co

Recibido: 13/12/2013

Aceptado: 04/02/2014 


\title{
RESUMEN
}

Debido a la limitada disponibilidad de agua para satisfacer los requerimientos de las poblaciones, los beneficios nutricionales a los suelos agrícolas y la disminución del impacto al ambiente, las aguas residuales se presentan como una alternativa importante para satisfacer la demanda de este recurso hídrico. Esta investigación evaluó la potencialidad del reúso del efluente de la Planta de Tratamiento de Aguas Residuales de Cañaveralejo, PTAR-C (Cali, Colombia) para el riego de caña de azúcar, empleando dos calidades de efluente tratado bajo las modalidades de tratamiento primario convencional (TPC) y avanzado (TPA) y agua de pozo como control. Aunque el análisis estadístico de los resultados mostró que no existió diferencia significativa en los resultados de las variables de respuesta en los tratamientos evaluados, se resalta el hecho que al utilizar el efluente de la PTAR-C en el riego de caña de azúcar se estaría evitando la descarga de alrededor de 43 toneladas de $\mathrm{DBO}_{5}$, 21 toneladas de SST y 9 toneladas de por día al río Cauca, lo que se reflejaría en una disminución de la contaminación de este cuerpo hídrico receptor y adicionalmente, se disminuiría la presión sobre los recursos hídricos empleados para el riego de este cultivo.

Palabras clave: Aguas residuales, agricultura, caña de azúcar, riego, reutilización.

\begin{abstract}
Many areas of the world face limited water availability to satisfy the population needs. It is widely recognized that treated wastewater can be reused as a source of nutrients in agricultural soil. This practice reduces the environmental impact by the discharge of these effluents in surface waters as well as the water resources demand for agricultural irrigation purposes. In this paper we evaluate the reuse potential of the effluent of the Cañaveralejo wastewater treatment plant WWTP-C for irrigation of soil used in sugar cane cultivation. Effluents from conventional primary treatment (CPT) and chemical enhanced primary treatment (CEPT) were used in the evaluation. The control was well water. Although the statistical analysis of the results not showed significant difference between the evaluated respond variables, the use of the treated wastewater would result in the reduction of the discharge to the Cauca River of 43 tons per day of Biological Oxygen Demand $\left(\mathrm{BOD}_{5}\right), 21$ tons per day of Total Suspended Solids (TSS) and 9 tons per day of. At the same time it would reduce the pressure on the water resources by the sugar cane cultivation in the region.
\end{abstract}

Keywords: Agriculture, irrigation, reuse, sugar cane, wastewater 


\section{INTRODUCCIÓN}

A nivel mundial, el 70\% del agua superficial y subterránea es empleada por el sector agrícola para riego, $20 \%$ se usa en la industria y $10 \%$ en consumo humano y doméstico (Shuval, 1990). Este hecho ha llevado a diferentes problemas de escasez de agua, por lo cual se han explorado nuevas alternativas para mejorar el suministro de este recurso.

La reutilización de aguas residuales es un concepto que ha incrementado su aceptación tanto en países industrializados como en países en desarrollo, debido principalmente al aumento de la población, la contaminación de los cuerpos de agua superficial y subterránea, la distribución desigual del recurso hídrico y los graves períodos secos que obligan a buscar nuevas fuentes de agua como las aguas residuales, principalmente para riego de cultivos.

Una de las prácticas más comunes de disposición final de las aguas residuales domésticas ha sido el vertimiento directo, sin tratamiento, a los cuerpos hídricos superficiales y al suelo. Sin embargo, la calidad de estas aguas puede generar problemas de salud pública por la alta incidencia de enfermedades infecciosas (Mara, 1996), así como problemas ambientales por afectar los ecosistemas acuáticos y el suelo, contribuyendo entre otras cosas, a la pérdida de valor económico del recurso y generando a su vez una disminución del bienestar para la comunidad ubicada aguas abajo de las descargas (Turner, Pearce y Bateman, 1993).

Son muchos los países, tanto industrializados como en desarrollo, que utilizan aguas residuales domésticas para regar suelos agrícolas (Madera, 2003). Mundialmente se ha calculado que el $18 \%$ de las tierras de cultivos son irrigadas con aguas residuales, produciendo el $40 \%$ de todos los alimentos. En el año 2004 alrededor de 20 millones de hectáreas en 50 países eran regados con aguas residuales crudas o parcialmente tratadas (Scott, Faruqui y Raschid, 2004). Sólo para el caso de Pakistán los agricultores que emplean las aguas residuales para riego obtienen alrededor de 300 dólares más por año que los agricultores que emplean otros tipos de agua para riego (WHO, 2006).

Esta investigación evaluó el efecto de la aplicación del efluente de la Planta de Tratamiento de Aguas Residuales de Cañaveralejo (PTAR-C) de la ciudad de Cali en las propiedades fisicoquímicas, microbiológicas y parasitológicas del suelo cultivado con caña de azúcar y en el rendimiento de este cultivo. 


\section{METODOLOGÍA}

La PTAR-C funciona como una planta de tratamiento primario, que puede operar bajo las modalidades de tratamiento primario convencional (TPC) o avanzado (TPA), este último consistente en la aplicación de cloruro férrico como coagulante y del polímero poliacrilamida aniónica como ayudante de floculación, con el objetivo de aumentar las eficiencias en remoción de Sólidos Suspendidos Totales (SST) y Demanda Bioquímica de Oxígeno $\left(\mathrm{DBO}_{5}\right)(\mathrm{EMCALI}, 2005)$. En la línea de agua (tratamiento preliminar y sedimentación primaria) se tratan las aguas residuales provenientes del colector central y las estaciones de bombeo Aguablanca, Cañaveralejo y Navarro; el efluente tratado es descargado al río Cauca y los lodos generados son conducidos a la línea de lodos para su tratamiento. Los caudales de diseño son $7.6 \mathrm{~m}^{3} / \mathrm{s}$ como caudal medio diario y $12.24 \mathrm{~m}^{3} / \mathrm{s}$ como caudal máximo (EMCALI, 2007).

Se emplearon tres calidades de agua de riego: i) Agua de pozo, ii) Agua residual tratada por TPA, iii) Agua residual tratada por TPC. En el valle del río Cauca se cuenta con un total de 1980 pozos explotados, de los cuales se emplean 1337 para uso agrícola (Torres, Cruz y Villegas, 2003) por lo tanto, se empleó agua de pozo como testigo con el objetivo de evaluar la influencia del uso de agua residual tratada en el rendimiento del cultivo de caña.

Como variables de control a las tres calidades de agua de riego se midieron parámetros fisicoquímicos, microbiológicos y parasitológicos durante los 10 meses de riego del cultivo de caña de azúcar. Las variables analizadas se muestran en la Tabla 1. 


\begin{tabular}{|c|c|c|c|}
\hline VARIABLE* & UNIDAD & $\begin{array}{l}\text { NÚMERO DE } \\
\text { MUESTRAS }\end{array}$ & TÉCNICA DE MEDICIÓN \\
\hline \multicolumn{4}{|l|}{ Fisicoquímico } \\
\hline DQO Total & $\mathrm{mg} / \mathrm{l}$ & 42 & Digestión titulación \\
\hline $\mathrm{DBO}_{5}$ & $\mathrm{mg} / \mathrm{l}$ & 42 & Oximetría \\
\hline Sólidos Totales -ST & $\mathrm{mg} / \mathrm{l}$ & 42 & Gravimétrica \\
\hline Conductividad & $\mathrm{dS} / \mathrm{m}$ & 42 & Potenciométrico \\
\hline NTK & $\mathrm{mgN} / \mathrm{l}$ & 11 & Digestión titulación \\
\hline $\mathrm{N}$ amoniacal & $\mathrm{mg} / \mathrm{l}$ & 11 & Titulométrico \\
\hline Nitratos $\mathrm{NO}_{3}$ & $\mathrm{mg} \mathrm{NO}_{3} / \mathrm{l}$ & 11 & Digestión titulación \\
\hline Nitritos $\mathrm{NO}_{2}$ & $\mathrm{mg} \mathrm{NO}_{2} / 1$ & 11 & Digestión titulación \\
\hline Fósforo Total & $\mathrm{mg} \mathrm{P} / \mathrm{l}$ & 11 & Digestión titulación \\
\hline Ortofosfatos & $\mathrm{mg} / \mathrm{l}$ & 11 & Digestión titulación \\
\hline Potasio & $\mathrm{mg} / \mathrm{l}$ & 11 & Espectrofotometría \\
\hline Sodio & $\mathrm{me} / \mathrm{l}$ & 11 & Espectrofotometría \\
\hline Calcio & $\mathrm{me} / \mathrm{l}$ & 11 & Espectrofotometría \\
\hline Magnesio & me/l & 11 & Espectrofotometría \\
\hline Boro & $\mathrm{mg} / \mathrm{l}$ & 11 & Espectrofotometría \\
\hline \multicolumn{4}{|c|}{ Microbiológico y Parasitológico } \\
\hline Coliformes totales & $\mathrm{UFC} / \mathrm{ml}$ & 11 & Filtración por membrana \\
\hline E. Coli & $\mathrm{UFC} / \mathrm{ml}$ & 11 & Filtración por membrana \\
\hline Huevos de Helmintos & $\mathrm{HH} / 100 \mathrm{ml}$ & 11 & Bailinger (1979) modificado \\
\hline
\end{tabular}

Tabla 1. Variables analizadas a las muestras de agua de riego. * APHA, AWWA y WPFC (2005)

A los resultados obtenidos del análisis de la calidad de las aguas empleadas para el riego se realizaron comparaciones e inferencias estadísticas entre los valores medios para todas las variables analizadas.

Se realizó la prueba de Kolmogorov-Smirnov para determinar si los datos presentaban una distribución normal. Definida la normalidad, se empleó la prueba de Bartlett para demostrar la homogeneidad de varianzas. A los datos que no cumplieron el supuesto de normalidad, se aplicó la prueba no paramétrica de Kruskal Wallis para contrastar si existía alguna diferencia.

En los datos que cumplieron el supuesto de normalidad y de homogeneidad se aplicó el análisis de varianza (ANOVA) para contrastar si existía alguna diferencia entre las medias de los grupos. Cuando la ANOVA mostró que existía diferencia entre los tratamientos fue necesario aplicar una prueba 
post-ANOVA. Como prueba post-ANOVA se empleó la comparación múltiple de Tukey que permite examinar en forma simultánea comparaciones entre todos los pares de grupos.

El suelo usado para la siembra fue definido como un suelo con características vérticas. En el año 2006 CENICAÑA reportó que el 63.8\% de la caña de azúcar del Valle del Cauca estaba cultivada en este tipo de suelo, por lo tanto, la siembra del cultivo en esta zona de estudio mostraba representatividad de la siembra a escala real (CENICAÑA, 2006).

Los tratamientos evaluados fueron tres, los cuales se describen en la Tabla 2. El diseño experimental empleado fue de bloques al azar, empleando tres bloques y tres réplicas por tratamiento.

\begin{tabular}{|c|c|c|}
\hline TRATAMIENTO & TIPO DE SUSTRATO & AGUA DE RIEGO \\
\hline 1 & Vertisol & Agua residual tratada por Tratamiento Primario Convencional \\
\hline 2 & Vertisol & Agua residual tratada por Tratamiento Primario Avanzado \\
\hline 3 & Vertisol & Agua de pozo \\
\hline
\end{tabular}

Tabla 2. Tratamientos evaluados

Con el objetivo de evaluar la influencia de la calidad de las aguas en los sustratos se realizaron análisis de la calidad fisicoquímica y microbiológica de los suelos. La Tabla 3 muestra las variables evaluadas y su técnica de medición.

\begin{tabular}{|c|c|c|}
\hline VARIABLE & UNIDAD & TÉCNICA DE MEDICIÓN \\
\hline Fisicoquímico & $\%$ & Digestión titulación \\
\hline Materia Orgánica & $\%$ & Digestión titulación \\
\hline Nitrógeno Total Kjeldahl & $\mathrm{mg} \mathrm{P} / \mathrm{Kg}$ & Digestión titulación \\
\hline Fósforo & & Filtración por membrana \\
\hline Microbiológico y Parasitológico & UFC/g & Filtración por membrana \\
\hline Coliformes totales & UFC/g & Bailinger (1979) modificado \\
\hline E. coli & $\mathrm{HH} / \mathrm{g}$ &
\end{tabular}

Tabla 3. Variables evaluadas en el suelo y técnica de medición

Con el objetivo de determinar el efecto del uso de los efluentes de la PTAR-C sobre la calidad microbiológica de los sustratos de cultivo, se realizó el análisis estadístico de los datos de los coliformes totales y E. coli en los sustratos, conforme al diseño experimental empleado de bloques al azar. 
Para la siembra se emplearon semillas vegetativas de la variedad CC 8592 con $60 \mathrm{~cm}$ de longitud por ser la más empleada en el Valle del Cauca (70\% del área sembrada) (CENICAÑA, 2012). La cosecha se realizó a los 13 meses de cultivo y se analizaron las variables mostradas en la Tabla 4.

\begin{tabular}{|l|c|c|c|}
\hline \multicolumn{1}{|c|}{ VARIABLE } & UNIDAD & FRECUENCIA & TÉCNICA DE MEDICIÓN \\
\hline Rendimiento & $\mathrm{Kg} / \mathrm{m}^{2}$ & Final & Directo \\
\hline Sacarosa & $\%$ & Final & Volumétrico \\
\hline
\end{tabular}

Tabla 4. Variables analizadas al cultivo

El análisis estadístico de los datos de las variables de respuesta en el cultivo (rendimiento y sacarosa) se realizó conforme al diseño experimental empleado que fue de bloques al azar.

\section{RESULTADOS Y DISCUSIÓN}

La Tabla 5 muestra los valores medios y la desviación estándar de las variables analizadas a las tres calidades de agua de riego.

\begin{tabular}{|c|c|c|c|c|c|c|c|}
\hline $\begin{array}{c}\text { AGUA } \\
\text { Variable }\end{array}$ & $n^{1}$ & $\begin{array}{l}\text { POZO } \\
\text { Media }\end{array}$ & $\mathrm{DE}^{3}$ & $\begin{array}{c}\text { TPA } \\
\text { Media }\end{array}$ & $\mathrm{DE}^{3}$ & $\begin{array}{c}\text { TPC } \\
\text { Media }\end{array}$ & $\mathrm{DE}^{3}$ \\
\hline \multicolumn{8}{|l|}{ Fisicoquímico } \\
\hline $\mathrm{DQO}(\mathrm{mg} / \mathrm{l})$ & 42 & 33.14 & 10.0 & 442 & 85.8 & 458 & 98.10 \\
\hline $\mathrm{DBO}_{5}(\mathrm{mg} / \mathrm{l})$ & 42 & 9.19 & 1.46 & 243.2 & 53.2 & 241.8 & 60.72 \\
\hline Sólidos Totales (mg/l) & 42 & 133.9 & 32.5 & 433.8 & 176.3 & 444.7 & 169.2 \\
\hline Conductividad (dS/m) & 42 & 0.388 & 0.02 & 0.576 & 0.038 & 0.573 & 0.049 \\
\hline NTK (mg/mg N/l ) & 11 & 10.5 & 7.38 & 21.27 & 12.8 & 17.70 & 12.21 \\
\hline $\mathrm{N}-\mathrm{NH}_{3}(\mathrm{mg} / \mathrm{l})$ & 11 & 6.35 & 3.02 & 14.35 & 10.2 & 14.17 & 10.68 \\
\hline Nitratos $\left(\mathrm{mg} \mathrm{NO}_{3} / \mathrm{l}\right)$ & 11 & 1.1 & 1.34 & 3.2 & 3.8 & 2.9 & 3.61 \\
\hline Nitritos $\left(\mathrm{mg} \mathrm{NO}_{2} / \mathrm{l}\right)$ & 11 & 0.058 & 0.02 & 0.053 & 0.031 & 0.042 & 0.021 \\
\hline Fósforo total (mg P/l) & 11 & 7.66 & 4.79 & 13.96 & 7.84 & 10.75 & 5.58 \\
\hline Ortofosfatos (mg /l) & 11 & 2.98 & 1.63 & 5.33 & 3.25 & 6.39 & 3.73 \\
\hline Potasio (mg/l) & 11 & 7.64 & 1.25 & 8.73 & 2.06 & 7.88 & 1.58 \\
\hline Sodio(me/l) & 11 & 1.77 & 1.26 & 1.51 & 1.05 & 1.30 & 0.97 \\
\hline Calcio(me/l) & 11 & 0.59 & 0.38 & 0.99 & 0.52 & 0.95 & 0.52 \\
\hline
\end{tabular}




\begin{tabular}{|c|c|c|c|c|c|c|c|}
\hline $\begin{array}{c}\text { AGUA } \\
\text { Variable }\end{array}$ & $\mathbf{n}^{1}$ & $\begin{array}{l}\text { POZO } \\
\text { Media } \\
\end{array}$ & $\mathrm{DE}^{3}$ & $\begin{array}{c}\text { TPA } \\
\text { Media } \\
\end{array}$ & $\mathrm{DE}^{3}$ & $\begin{array}{c}\text { TPC } \\
\text { Media } \\
\end{array}$ & $\mathrm{DE}^{3}$ \\
\hline \multicolumn{8}{|l|}{ Fisicoquímico } \\
\hline Magnesio (me/l) & 11 & 1.21 & 1.21 & 1.20 & 1.17 & 1.09 & 0.99 \\
\hline $\mathrm{RAS}^{2}$ & 11 & 2.15 & 1.63 & 1.55 & 1.11 & 1.40 & 1.12 \\
\hline Boro (ppm) & 11 & 0.0158 & 0.007 & 0.041 & 0.016 & 0.033 & 0.017 \\
\hline \multicolumn{8}{|c|}{ Microbiológica y Parasitológica } \\
\hline $\begin{array}{l}\text { Coliformes totales } \\
\text { (UFC/100ml) }\end{array}$ & 11 & $4.59 \times 10^{4^{\circ}}$ & $1.25 \times 10^{5}$ & $2.12 \times 10^{7 *}$ & $5.74 \times 10^{7}$ & $1.61 \times 10^{7 *}$ & $\begin{array}{c}1.03 \mathrm{x} \\
10^{7}\end{array}$ \\
\hline E. Coli (UFC/100ml) & 11 & $1.44 \times 10^{3^{3}}$ & $3.28 \times 10^{3}$ & $4.98 \times 10^{6 *}$ & $1.38 \times 10^{7}$ & $3.64 \times 10^{6 *}$ & $\begin{array}{c}3.95 x \\
10^{6}\end{array}$ \\
\hline $\begin{array}{c}\text { Huevos de Helmintos } \\
(\mathrm{HH} / \mathrm{l})\end{array}$ & 11 & $<1$ & - & 15 & 27 & 12 & 19 \\
\hline
\end{tabular}

Tabla 5. Caracterizaciones de las aguas de riego ${ }^{1} \mathrm{n}$ : Cantidad total de datos ${ }^{2}$ Relación Adsorción de Sodio-RAS ${ }^{3} \mathrm{DE}$ : Desviación Estándar *Media geométrica

Los valores de las variables analizadas al agua de pozo se encuentran dentro de los niveles normales de este tipo de aguas, presentando valores de $\mathrm{pH}$ en el rango neutro, bajos contenidos de materia orgánica e inorgánica, sólidos, nutrientes y metales como hierro y aluminio. Así mismo, presenta concentraciones altas de sodio, magnesio y bicarbonatos característicos de estas aguas ya que éstos son aportados por las rocas presentes en el suelo.

El agua de pozo empleada no presenta restricción de uso en la agricultura y es clasificada como un agua clase C2S1 (USDA, 1954), que significa salinidad media y bajo contenido de sodio, apta para el riego, pero puede presentar problemas en cultivos sensibles al sodio. Desde el punto de vista microbiológico, esta agua presenta una concentración mucho más baja de coliformes totales y E. coli (alrededor de 3 unidades logarítmicas) que los otros dos tipos de agua residual tratada y con un riesgo más bajo de infección.

Las características de los dos efluentes de la PTAR-C empleados para el riego permiten definirlas como aguas residuales sin ningún grado de restricción para uso agrícola desde el punto de vista fisicoquímico y son clasificadas como clase C2S1 con una salinidad media y bajo contenido de sodio, apta para el riego según el USDA (1954). Sin embargo, los valores de sólidos suspendidos, fósforo total y bicarbonato no cumplen las recomendaciones de la norma de calidad de riego para la agricultura de la FAO (Ayers y Westcot, 1985). Los sólidos suspendidos son de gran importancia principalmente en los sistemas de riego de los cultivos ya que pueden ocasionar obstrucciones que afectarían dichos sistemas, el fósforo suple los requerimientos de los cultivos y los niveles elevados no causan ningún efecto en la planta; pero éste puede causar problemas al drenar a los cuerpos de aguas superficiales (USEPA, 2004). 
Las variables microbiológicas y parasitológicas de las aguas residuales tratadas presentan restricción en su uso por los valores altos de coliformes totales ( $2.12 \times 10^{7} \mathrm{TPA}$ y $\left.1.6 \times 10^{7} \mathrm{TPC}\right)$, E. Coli $(4.98 \mathrm{x}$ $10^{6}$ TPA y 3.64 x $10^{6}$ TPC) y huevos de helmintos (15 TPA y 12 TPC) según las directrices de calidad de la OMS para riego (WHO, 2006). Por lo tanto, para el uso de estas aguas es necesario identificar los diversos patógenos presentes para definir los periodos de sobrevivencia en el suelo y en los cultivos y establecer medidas de protección y control en el campo de cultivo y en los agricultores, acciones como el uso de ropa gruesa, botas y guantes y el tiempo de cosecha después del último riego son medidas que permiten minimizar el riesgo a la salud. Se observa que el sistema de tratamiento no está eliminando estos microorganismos, solamente alrededor de 1 unidad logarítmica, lo que está de acuerdo con las remociones reportadas para un tratamiento primario.

La concentración de nitrógeno, fósforo, potasio y boro en las aguas residuales, muestra la potencialidad de su uso en algunos cultivos como forrajes perennes, ciertas especies arbóreas, cultivos como el maíz, el sorgo, la cebada y algunos cultivos frutales como los cítricos (Metcalf y Eddy, 2003). La principal forma de nitrógeno presente es el nitrógeno amoniacal (casi el 60\% de NTK), condición esperada con el nivel de tratamiento de las aguas residuales.

El análisis estadístico de los datos mostró que todos los tratamientos presentaron normalidad en la distribución y homogeneidad de varianza. La ANOVA mostró que las variables nitratos (grados de libertad- $g l=2, p=0.144)$, fósforo $(g l=2, p=0.074)$, sodio $(g l=2, p=0.608)$, calcio $(g l=2, p=0.092)$ y la relación de absorción de sodio - RAS $(g l=2, p=0.384)$ no presentan diferencia significativa para las tres calidades agua de riego. Adicionalmente, como las otras variables mostraron que existe diferencia entre las tres calidades de agua, fue necesario realizar la prueba post-ANOVA (Tukey) para definir en cuál de las aguas de riego se presenta diferencia o igualdad.

La prueba post-ANOVA mostró que no existen diferencias significativas entre los valores medios presentados por todas las variables evaluadas a las dos calidades de agua residual tratada. En relación con el agua de pozo, una parte de las variables evaluadas presentan diferencia significativa con las dos calidades de agua residual tratada, con excepción de los nitratos, fósforo, sodio, calcio, magnesio y RAS, lo que indica que para las tres calidades de agua no se presentarían problemas de salinidad e infiltración si se emplean en la agricultura.

La Tabla 6 muestra los resultados fisicoquímicos, microbiológicos y parasitológicos del suelo al final del proceso del cultivo irrigado con los tres tipos de agua empleados en el estudio. 


\begin{tabular}{|c|c|c|c|c|}
\hline VARIABLE* & POZO & TPA & TPC \\
\hline Fisicoquímico & UNIDADES & \multicolumn{3}{l|}{} \\
\hline Materia Orgánica & $\%$ & 3.0 & 2.6 & 4.1 \\
\hline Nitrógeno Total Kjeldahl & $\%$ & 0.15 & 0.13 & 314.8 \\
\hline Fósforo Total & $\mathrm{mg} \mathrm{P} / \mathrm{kg}$ & 302.8 & 361.9 & $5.94 \times 10^{6}$ \\
\hline Microbiológico y Parasitológico & & & \\
\hline Coliformes totales & UFC/g & $2.31 \times 10^{6}$ & $4.15 \times 10^{6}$ & $8.17 \times 10^{4}$ \\
\hline E. coli & UFC/g & $8.89 \times 10^{4}$ & $7.56 \times 10^{4}$ & $<1$ \\
\hline
\end{tabular}

Tabla 6. Resultados fisicoquímicos, microbiológicos y parasitológicos del suelo al final del cultivo. TPA: Agua residual tratada por Tratamiento Primario Avanzado, TPC: Agua residual tratada por Tratamiento Primario Convencional

En la parte plana del valle del río Cauca predominan suelos con contenidos de materia orgánica menores a 4\% (Quintero, 1993); todos los resultados encontrados mostraron valores al final del cultivo (mes 12) menores con excepción del tratamiento irrigado con agua residual del tratamiento primario convencional-TPC el cual fue mayor, evidenciando que en este sustrato el uso de agua residual permitió probablemente que se presentaran procesos de humificación de la materia orgánica lo que se evidenció en el aumento de la misma en este tratamiento. El análisis estadístico mostró que el uso de las aguas residuales no influye significativamente $(g l=2, p=0.727)$ en el contenido de la materia orgánica del suelo a los 12 meses del cultivo.

El nitrógeno es un nutriente esencial para el crecimiento de los vegetales, ya que es un constituyente de todas las proteínas. Los valores en los suelos pueden variar entre 0.2 al $0.7 \%$ (Quintero, 1993) y los resultados obtenidos en todos los tratamientos fueron menores a este rango mostrando que el uso de las aguas residuales no influye significativamente $(g l=2, p=0.708)$ en el contenido del nitrógeno total al final del cultivo.

La mayoría de los suelos de la parte plana del valle del río Cauca presentan contenidos de fósforo disponible superiores a $10 \mathrm{mg} / \mathrm{kg}$. Todos los tratamientos mostraron valores superiores, lo que hace suponer que estos sustratos suplieron las necesidades de dicho elemento en el cultivo. Sin embargo, el análisis estadístico mostró que no existe diferencias significativas $(g l=2, p=0.695)$ entre los tratamientos evaluados, lo que muestra que el uso de aguas residuales no influencia esta variable para los tratamiento evaluados. 
Con base en las investigaciones realizadas con caña de azúcar en suelos del valle del río Cauca, se estableció que valores menores a $0.15 \mathrm{meq} / 100 \mathrm{~g}$ presentan niveles críticos para el potasio intercambiable. Todos los tratamientos presentaron valores superiores a este nivel crítico. Sin embargo, el análisis estadístico no mostró diferencias significativas $(g l=2, p=0.887)$ entre los tratamientos evaluados.

El comportamiento de las variables microbiológicas en los sustratos mostró que el uso de cualquiera de la calidades de agua de riego empleadas en la investigación tienen un efecto en las variables microbiológicas de los sustratos, que se evidenció en el alto contenido de coliformes totales y E. Coli al finalizar el cultivo, lo que representa un riesgo bajo a los agricultores. Esto demuestra la necesidad de implementar medidas de protección, principalmente en los momentos que se está en contacto con el cultivo. Sin embargo, el análisis estadístico de las variables microbiológicas evidenció que no existe diferencia significativa entre los valores medios de los tratamientos; lo que muestra que el usar aguas residuales tratadas por TPA o TPC o agua de pozo para riego en caña no representa una diferencia significativa en el comportamiento de los coliformes totales $(g l=2, p=0.054)$ y E. Coli $(g l=2, p=0.994)$ en los sustratos.

La Tabla 7 muestra los resultados de rendimiento del cultivo y sacarosa en el jugo extraído de la caña de azúcar.

\begin{tabular}{|c|c|c|}
\hline AGUA & RENDIMIENTO $\left(\mathbf{k g} / \mathbf{m}^{2}\right)$ & SACAROSA $(\% \mathbf{m} / \mathbf{v})$ \\
\hline Pozo & 12.17 & 1.8 \\
\hline TPA & 10.4 & 6.3 \\
\hline TPC & 13.33 & 4.1 \\
\hline
\end{tabular}

Tabla 7. Resultados del rendimiento y sacarosa

Los valores de productividad fueron similares para todos los tratamientos, lo que se corroboró en el análisis estadístico de los datos, donde no hubo diferencias significativas entre los tratamientos $(g l=2, p=0.839)$. Así mismo, se encontró que el contenido de sacarosa en el jugo de la caña de azúcar se incrementó en los tratamientos irrigados con agua residual. Sin embargo, el análisis estadístico de los datos no mostró diferencias significativas entre los tratamientos ( $g l=2, p=0.27$ ).

Aunque el análisis estadístico de los resultados mostró que no existe una diferencia significativa en los mismos para las variables de respuesta de los tratamiento evaluados, vale la pena resaltar el importante aporte en la mitigación del impacto ambiental si el efluente de la PTAR-C se empleara en el riego de caña de azúcar, ya que se estaría evitando la descarga de alrededor de 43 toneladas de $\mathrm{DBO}_{5}, 21$ toneladas de SST y 9 toneladas de $\mathrm{NH}_{3}$ por día lo que se reflejaría en una disminución de 
la contaminación del río Cauca. Adicionalmente, se disminuiría la presión en los recursos hídricos empleados para el riego de caña, ya que con el caudal actual de operación de la PTAR-C se podría irrigar alrededor de 12250 hectáreas lo que correspondería al 5.5\% del área total de caña sembrada para el 2011 en el Valle del Cauca (CENICAÑA, 2012).

En relación con el contenido de nutrientes presentes en efluente de la PTAR-C se calculó la carga aplicada a cada tratamiento teniendo en cuenta el volumen aplicado de $0.712 \mathrm{~m}^{3}$ y las características químicas de los efluentes. La carga de nutrientes aplicada a cada tratamiento con las aguas de riego se muestra a continuación:

\begin{tabular}{|c|c|c|c|}
\hline \multirow{2}{*}{ NUTRIENTE $\left(\mathrm{Kg} / \mathrm{m}^{2}\right)$} & \multicolumn{3}{|c|}{ AGUA DE RIEGO } \\
\cline { 2 - 4 } & \multirow{2}{*}{ POZO } & TPA & TPC \\
\hline Nitrógeno & 0.0150 & 0.0303 & 0.0252 \\
\hline Fosforo & 0.0109 & 0.0199 & 0.0153 \\
\hline Potasio & 0.0109 & 0.0124 & 0.0112 \\
\hline
\end{tabular}

Tabla 8. Carga de nutrientes aplicados a cada tratamiento en $\mathrm{Kg} / \mathrm{m}^{2}$

La Tabla 8 muestra que las dos calidades de agua residual tratada (TPA y TPC) empleadas aportaron valores mayores de nutrientes al cultivo de caña de azúcar en comparación con el agua de pozo, ya que superaron el contenido de nitrógeno aplicado de 68 al 102\% y de fósforo de 40 al 82.6\%. Así mismo, con las dos calidades de agua residual tratada se aportan nutrientes adicionales a las necesidades del cultivo según lo recomendado por Aoorenbos y Kassan, (1979) (de 100 a $200 \mathrm{~kg} / \mathrm{Ha}$ de N, de 20 a 90 $\mathrm{kg} / \mathrm{Ha}$ de $\mathrm{P}$, y de 125 a $160 \mathrm{~kg} / \mathrm{Ha}$ de $\mathrm{K}$ ), con excepción del potasio que se encuentra por debajo de dichas recomendaciones. Adicionalmente, se evidencia la alta potencialidad de reutilización de dichos efluentes ya que se presentaría una reducción de los costos de fertilización, pues solo sería necesario adicionar potasio para suplir el contenido que no es aportado por las aguas residuales.

\section{CONCLUSIONES}

Los efluentes obtenidos en las dos modalidades de operación Tratamiento Primario Avanzado-TPA y Tratamiento Primario Convencional-TPC y el agua pozo, son potencialmente utilizables para el riego de caña de azúcar desde el punto de vista de la calidad agronómica (Ayers y Westcot, 1985; USDA, 1954), no presentan restricción de uso de acuerdo con las directrices internacionales. Sin embargo, presentan restricción en su uso para riego agrícola en cultivos de consumo directo por las concen- 
traciones altas de coliformes totales $\left(2.12 \times 10^{7}\right.$ TPA y $\left.1.6 \times 10^{7} \mathrm{TPC}\right)$, E. Coli ( $4.98 \times 10^{6}$ TPA y $3.64 \mathrm{x}$ $10^{6}$ ), y huevos de helmintos (15 TPA y 12 TPC) según las directrices de calidad de la OMS (2006). Por lo tanto, para el uso de este tipo de aguas en el cultivo de caña de azúcar es necesario establecer medidas de protección y control al agricultor como el uso de ropa gruesa, botas y guantes principalmente en los momentos que se está en contacto con la planta y el suelo así como en la realización de los riegos y la cosecha.

Los valores de rendimiento del cultivo fueron similares para los tratamientos evaluados, sin embargo, el contenido de sacarosa en el jugo extraído mejoró con el riego de aguas residuales comparado con el cultivo irrigado con agua de pozo, lo que evidencia el alto potencial de uso de los efluentes de la PTAR-C y teniendo en cuenta que la carga de nitrógeno superó en un 68 al 102\% comparado con el agua de pozo se muestra su alto potencial de aprovechamiento para riego agrícola. 


\section{REFERENCIAS BIBLIOGRÁFICAS}

Aoorenbos, J. y Kassan, A. H. (1979). Efectos del agua sobre el rendimiento de los cultivos. Organización de las naciones unidas para la agricultura y la alimentación-FAO. Serie Riego y drenaje №33. Roma, 212p.

APHA, AWWA y WPFC. (2005). Standard Methods for the Examination of Waters and Wastewaters. 21th Edition. Estados Unidos. 1200p.

Ayers, R.S y Westcot, D.W. (1985). Water quality for agriculture. Irrigation and drainage. Technical paper № 29. Food and Agriculture Organization of the United Nations, FAO. Rome. 174p.

Bailinger, J. (1979). Mechanisms of parasitological concentration in coprology and their practical consequences. The American Journal of Medical Technology (41), pp: 65-71.

CENICAÑA- Centro de Investigación de la Caña. (2012). Informe anual 2011. Cali. 136p.

CENICAÑA- Centro de Investigación de la Caña-. (2006). Carta trimestral. Producción de caña de azúcar en el valle del Río Cauca. Año 28.№1. 2006. 37p.

EMCALI. (2007). Informe ejecutivo de operación año 2006. Gerencia de acueducto y alcantarillado. Cali. 100p.

EMCALI. (2005). Informe de descargas finales al río Cauca. Gerencia de acueducto y alcantarillado. Cali. 98p.

Mara, D. (1996). Waste Stabilization Ponds: Effluent quality requirements and implications for process design. Water Science and Technology (33), 23-31.

Madera, C. (2003). Microbiological and agronomic effluent quality from duckweed and stabilization ponds. Ginebra, Colombia. Msc Thesis. Institute for Water Education The Netherlands, UNESCO-IHE. 87P.

Metcalf y Eddy Inc. (2003). Waste engineering: treatment, and reuse. Fourth Edition Ed. Mc Graw Hill. 1819p.

Turner K., Pearce D. y Bateman, I. (1993). Economics resources and the environment. Jhon Hopkins University Press. Baltimore. 34p.

Quintero, R. (1993). Interpretación del análisis de suelo y recomendaciones de fertilizantes para la caña de azúcar. Centro de investigación de la caña de azúcar de Colombia-CENICAÑA. Serie Técnica No. 14.17p. 
Scott, C.; Faruqui, N.I. y Raschid, L. (2004). Wastewater use in irrigated agriculture: confronting the livelihood and environmental realities. CABI, IWMI, IDRC. 206p.

Shuval, H. I. (1990). Health effects of wastewater irrigation and their control in developing countries. The World Bank, integrated resource recovery project series number GLO/80/004. 340 p.

Torres, J; Cruz, R; Villegas, F. (2003) Manejo del riego en la caña de azúcar. XV congreso ATACORI. Asociación de técnicos azucareros de Costa Rica. Costa Rica. 81p.

USDA-United State Department Of Agricultural. (1954). Diagnosis and improvements of saline and alkali soils. Agriculture Handbook № 60, United States Department of Agriculture, Washington, D.C. 160 p.

USEPA (U. S. Environmental Protection Agency), (2004).Guidelines for Water Reuse. Technology Transfer and Support Division National Risk Management Research Laboratory Office of Research and Development Cincinnati, O. 245p

WHO. (2006). World Health Organization. Guidelines for the safe use of wastewater excreta and greywater. Volume 2. Wastewater Use in Agriculture. 191p. 


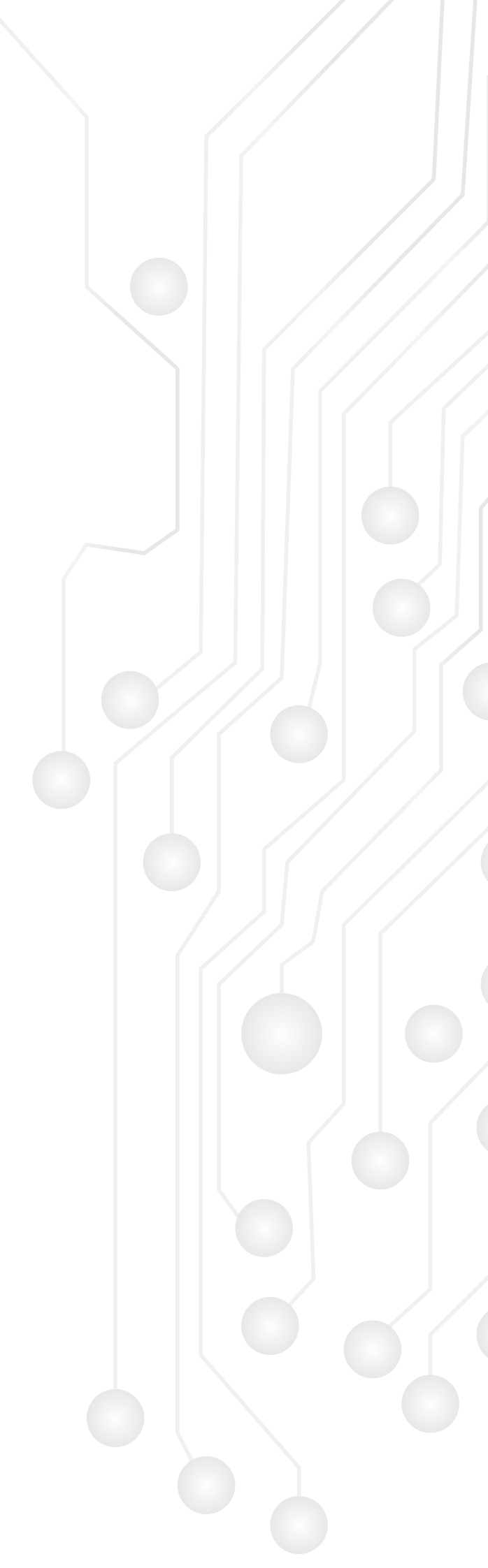

